\title{
Electrostatic correlations in colloidal suspensions: Density profiles and effective charges beyond the Poisson-Boltzmann theory
}

Cite as: J. Chem. Phys. 130, 124110 (2009); https://doi.org/10.1063/1.3098556

Submitted: 16 January 2009 . Accepted: 21 February 2009 . Published Online: 24 March 2009

Alexandre P. dos Santos, Alexandre Diehl, and Yan Levin

\section{ARTICLES YOU MAY BE INTERESTED IN}

Effects of the dielectric discontinuity on the counterion distribution in a colloidal suspension

The Journal of Chemical Physics 135, 044124 (2011); https://doi.org/10.1063/1.3615940

Charge renormalization, osmotic pressure, and bulk modulus of colloidal crystals: Theory

The Journal of Chemical Physics 80, 5776 (1984); https://doi.org/10.1063/1.446600

Colloidal charge renormalization in suspensions containing multivalent electrolyte

The Journal of Chemical Physics 132, 104105 (2010); https://doi.org/10.1063/1.3354120

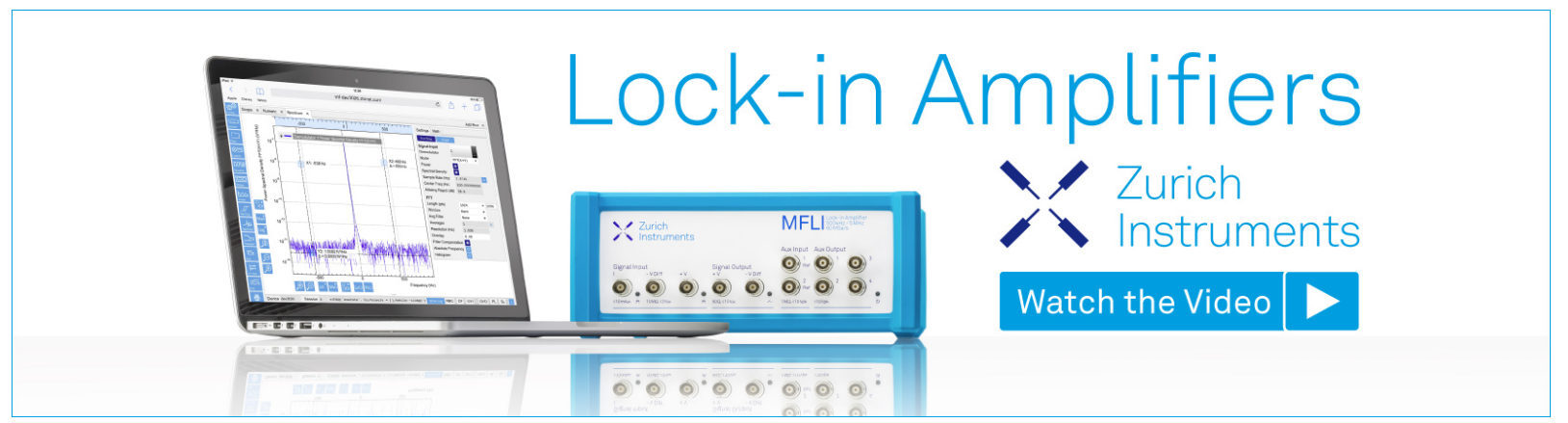

J. Chem. Phys. 130, 124110 (2009); https://doi.org/10.1063/1.3098556 


\title{
Electrostatic correlations in colloidal suspensions: Density profiles and effective charges beyond the Poisson-Boltzmann theory
}

\author{
Alexandre P. dos Santos, ${ }^{1}$ Alexandre Diehl, ${ }^{2}$ and Yan Levin ${ }^{1, a)}$ \\ ${ }^{1}$ Instituto de Física, Universidade Federal do Rio Grande do Sul, Caixa Postal 15051, \\ CEP 91501-970 Porto Alegre, RS, Brazil \\ ${ }^{2}$ Departamento de Física, Instituto de Física e Matemática, Universidade Federal de Pelotas, \\ Caixa Postal 354, CEP 96010-900 Pelotas, RS, Brazil
}

(Received 16 January 2009; accepted 21 February 2009; published online 24 March 2009)

\begin{abstract}
A theory is proposed which allows us to calculate the distribution of the multivalent counterions around a colloidal particle using the cell model. The results are compared with the Monte Carlo simulations and are found to be very accurate in the two asymptotic regimes, close to the colloidal particle and far from it. The theory allows to accurately calculate the osmotic pressure and the effective charge of colloidal particles with multivalent counterions. (C) 2009 American Institute of Physics. [DOI: 10.1063/1.3098556]
\end{abstract}

\section{INTRODUCTION}

It is well known that Poisson-Boltzmann (PB) theory is very accurate in predicting the distributions of counterions and coions inside the aqueous suspensions containing 1:1 electrolyte. ${ }^{1}$ Unfortunately this good agreement disappears quickly when besides simple 1:1 salt, suspensions also contain multivalent ions. ${ }^{2,3}$ In these cases the PB theory fails dramatically. It predicts that for strongly charged colloidal particles, the counterion and coion density profiles saturate, becoming completely independent of the colloidal charge. ${ }^{4}$ This saturation is also reflected in the value of the effective colloidal charge which is found to be independent of the bare charge for strongly charged particles. ${ }^{5,6}$ This is contrary to the results of Monte Carlo (MC) and molecular dynamics simulations, which found that the effective colloidal charge in suspensions with multivalent counterions reaches a maximum and then decreases as a function of the colloidal bare charge. ${ }^{7,8}$ The density profiles also do not show the saturation effects predicted by the $\mathrm{PB}$ theory and are found to strongly depend on the bare colloidal charge. ${ }^{9,10}$ The nonmonotonic dependence of the effective colloidal charge on the bare charge leads to some very counterintuitive behavior. For example, it has been observed experimentally that the mobility of colloids can become reversed as a function of the multivalent electrolyte concentration. ${ }^{11,12}$ A related phenomenon is the attraction between like charged colloidal particles, also found in suspensions containing multivalent counterions. ${ }^{13,14}$ Both phenomena are the result of strong positional correlations between the multivalent counterions. ${ }^{1,12,15}$ These correlations are completely unaccounted for within the PB theory. Although qualitatively the two phenomena are now well understood, ${ }^{1,12,16-18}$ there is a distinct lack of quantitative theories which can be compared directly with the MC simulations. In this paper, we present one such theory for the special case of colloids with multivalent counterions in the absence of salt.

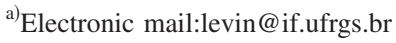

\section{THE MODEL}

To study the distribution of counterions and the effective colloidal charge, we use a cell model. A colloid of radius $a$ and charge $-Z q$ is placed at the center of a spherical WignerSeitz (WS) cell of radius $R$, determined by the concentration of particles inside the suspension. The cell also contains $Z / \alpha$ counterions of valence $\alpha$, charge $+\alpha q$, and radius $r_{c}$. The solvent is treated as a structureless medium of dielectric permittivity $\epsilon$. The macroion charged groups are considered to be uniformly distributed on the colloidal surface and are fully ionized. The Bjerrum length is defined as $\lambda_{B}=q^{2} / \epsilon k_{B} T$ and is fixed at $\lambda_{B}=7.2 \AA$, the value for water at room temperature, for all the results presented below. To test the theoretical predictions, all the results are compared with the canonical MC simulations, the details of which are given in Ref. 9.

In the case of monovalent counterions $(\alpha=1)$, the counterion distribution can be obtained very accurately by solving the PB equation:

$$
\nabla^{2} \phi(r)=\frac{Z q}{\epsilon a^{2}} \delta(r-a)-\frac{4 \pi}{\epsilon} \alpha q \rho(r) 0,
$$

where $\phi(r)$ is the electrostatic potential at distance $r$ from the colloidal center and the counterion density profile $\rho(r)$ is determined by the Boltzmann distribution:

$$
\rho(r)=\frac{Z \exp [-\beta \alpha q \phi(r)]}{4 \pi \alpha \int_{a}^{R} d r r^{2} \exp [-\beta \alpha q \phi(r)]} .
$$

Note that in the expression above enters the electrostatic potential and not the potential of mean force. Therefore, this equation is approximate, valid only at the mean-field level-as long as the electrostatic correlations between the counterions are negligible. Compared to the $\mathrm{MC}$ simulations, it is found that in the case of monovalent counterions the density profiles are very accurately described by the PB equation. ${ }^{9,10}$ However, if the suspensions contain multivalent 

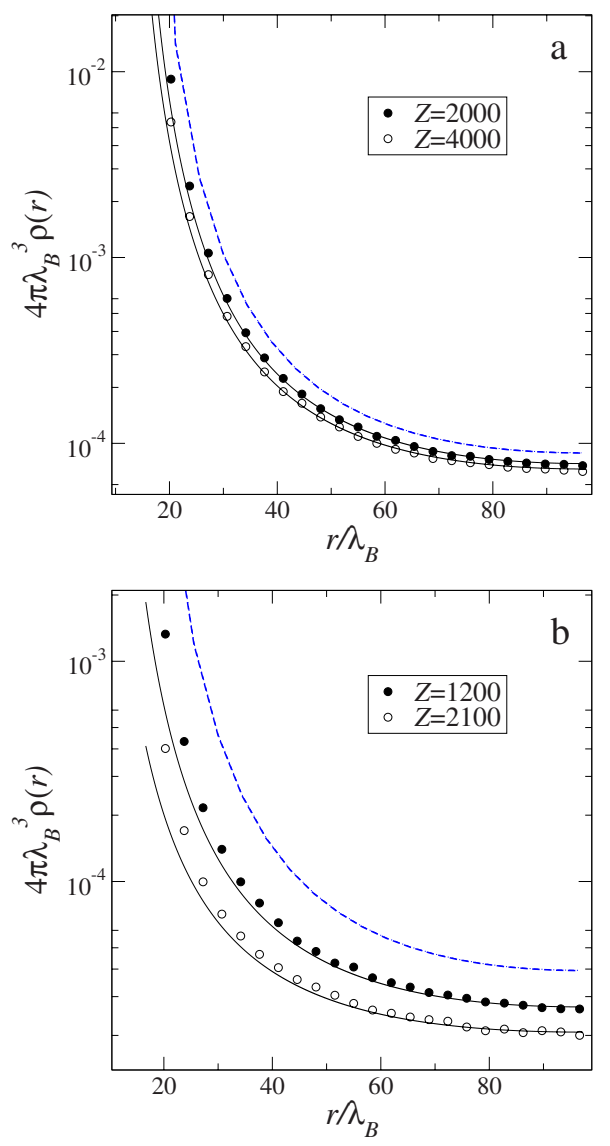

FIG. 1. (Color online) Counterion density profiles for (a) divalent and (b) trivalent counterions. The symbols are the simulation data and the solid lines obtained with the present theory. At short distance there is a crossover to strongly correlated regime described by Eq. (4). The dashed curve corresponds to the two values of $Z$ which overlap completely within the PB theory. It deviates strongly from the simulations. The counterion radius is 1 $\AA$, the colloidal radius is $120 \AA$, and the WS cell is $700 \AA$ for all the cases.

counterions, or the solvent is of low dielectric permittivity, the correlations between the counterions become important and the PB equation loses its accuracy. ${ }^{9,10}$ This is clearly demonstrated in Fig. 1 for the divalent and trivalent counterions.

For strongly charged colloids, the PB equation predicts the characteristic saturation of the density profiles; for two very different colloidal charges, the distributions of counterions after a short distance from the colloidal surface become completely indistinguishable, superimposing on one single curve, Fig. 1. This is clearly not the case in the MC simulations. Although for divalent counterions the difference between the two curves is not very large, it is quite noticeable-while the counterion density profiles deviate significantly from the PB prediction. For trivalent ions, the deviations become even stronger and the dependence on the bare colloidal charge much more pronounced. Clearly the PB theory cannot be used to accurately describe these systems. As an alternative one can use integral equations ${ }^{3,18,19}$ or weighted density functional theories, ${ }^{7,20,21}$ both of which, however, are much more complicated and more difficult to solve numerically, as well as a lot less transparent than the simple PB equation.

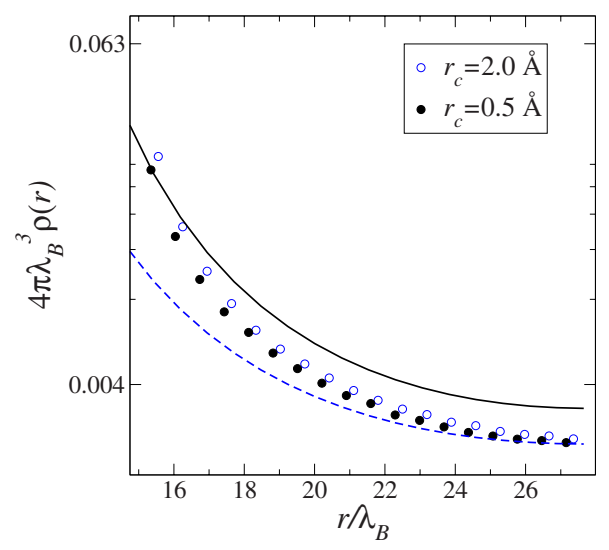

FIG. 2. (Color online) Divalent counterion distribution around a macroion of charge $Z=3000$, radius $a=100 \AA$ inside a WS cell of radius $R=200 \AA$, for two different ionic sizes. Note a very weak dependence of the counterion density distribution on the ionic radius observed in the MC simulations (symbols), while the matching procedure based on the ionic radius leads to a very strong variation in the density profiles with the ionic size (solid lines).

\section{THE THEORY}

An interesting approach to overcome the shortcomings of the PB theory was recently proposed by Shklovskii. ${ }^{17}$ Since far from the colloidal surface the density of counterions is small, the correlations between the counterions there should be insignificant. On the other hand, the correlations are very important near the colloidal surface, where the counterions form a strongly correlated fluid. Shklovskii argued that it should be possible to "match" the two regimes. He then suggested that the concentration of counterions inside the strongly correlated sheath should be approximated by $Z / 4 \pi a^{2} w$, where $w$ is the diameter of a water molecule. Unfortunately, it is difficult to see why the diameter of a water molecule should be the relevant scale for the width of the sheath containing strongly correlated counterions. Furthermore, if we want to compare the theory with the continuum dielectric models used in simulations, there is no associated water molecule length scale available. One can suppose that in this case $w$ should be replaced by the hydrated ionic radius. It is, however, easy to see that this kind of approach is bound to fail. If one tries to do the matching procedure using the ionic radius, the resulting density profiles will be strongly dependent on the ionic size. This, however, is not the case in the MC simulations, which show that the counterion density profiles are quite insensitive to the ionic size, see Fig. 2. In this paper, based on the contact theorem ${ }^{22}$ and the strong coupling expansion, ${ }^{23}$ we propose a different approach which gives an excellent agreement with the MC simulations.

The contact theorem ${ }^{22}$ can be used to relate the densities of the counterions at the cell boundary $\rho(R)$ and at the colloidal surface $\rho\left(a+r_{c}\right)$,

$$
\rho\left(a+r_{c}\right)=\rho(R)+\frac{Z^{2} \lambda_{B}}{8 \pi\left(a+r_{c}\right)^{4}} .
$$

In the case of multivalent counterions, the counterion concentration at the cell boundary is very low, so that Eq. (3) allows us to determine very accurately the average 


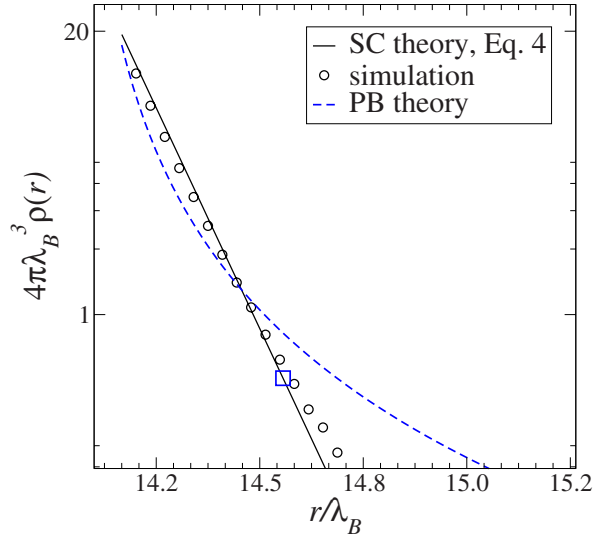

FIG. 3. (Color online) Counterion density profile close to the macroion surface: the solid line is the strong coupling theory Eq. (4), the dashed line is the solution of the PB equation, and the symbols are the result of the MC simulations; $Z=1200, a=100 \AA, \alpha=3, r_{c}=2 \AA$, and $R=200 \AA$. The square indicates the distance $r=a+r_{c}+l_{\mathrm{sc}}$ at which the simulations start to deviate from the strong coupling limit.

counterion concentration at the contact with colloid, $\rho\left(a+r_{c}\right)=Z^{2} \lambda_{B} / 8 \pi\left(a+r_{c}\right)^{4}$. Therefore, in the strong coupling limit ${ }^{15,23}$ the concentration of counterions in the vicinity of colloidal surface must vary as

$$
\rho(r)=\frac{Z^{2} \lambda_{B}}{8 \pi\left(a+r_{c}\right)^{4}} e^{-\left(r-a-r_{c}\right) / \lambda_{\mathrm{GC}}},
$$

where $\lambda_{\mathrm{GC}}=2 a^{2} / Z \alpha \lambda_{B}$ is the Gouy-Chapman length. This is an asymptotic result, valid in the limit when the Coulomb interaction between the condensed counterions is significantly larger than the thermal energy, $\alpha^{2} q^{2} / \epsilon d \gg k_{B} T$, where $1 / \pi d^{2}$ is the average two-dimensional (2D) density of condensed counterions on the colloidal surface. This condition can be conveniently expressed in terms of the traditional 2D plasma parameter $\Gamma$ as

$$
\Gamma \equiv \frac{\alpha^{3 / 2} \lambda_{B} \sqrt{Z}}{2\left(a+r_{c}\right)} \gg 1
$$

Compared with the MC simulations, we see that Eq. (4) describes perfectly the counterion concentration in the vicinity of the colloidal surface, Fig. 3. It also provides us with a natural length scale $l_{\mathrm{sc}}$ which delimits the region of the quasi-2D strongly correlated fluid of condensed counterions. This length scale corresponds to the distance at which Eq. (4) begins to deviate from the MC results, approximately $l_{\mathrm{sc}} \approx 3.6 \lambda_{\mathrm{GC}}$, as is shown by the square in Fig. 3 . The value of $l_{\mathrm{sc}}$ is found to be approximately the same for all the systems studied in this paper, independent of the colloidal size and the counterion radius. Within the distance $l_{\mathrm{sc}}$, the correlational effects dominate over the mean field, so that the expression (4) describes very accurately the counterion distribution. Further away, however, when $r>a+r_{c}+l_{\mathrm{sc}}$, the strong coupling distribution starts to deviate from the MC results.

Far from the colloidal surface, the correlations between the counterions become weak and the density distribution is governed by the mean-field PB equation. The difficulty resides in matching the two density profiles. Shklovskii and others argued that the condensed counterions close to the

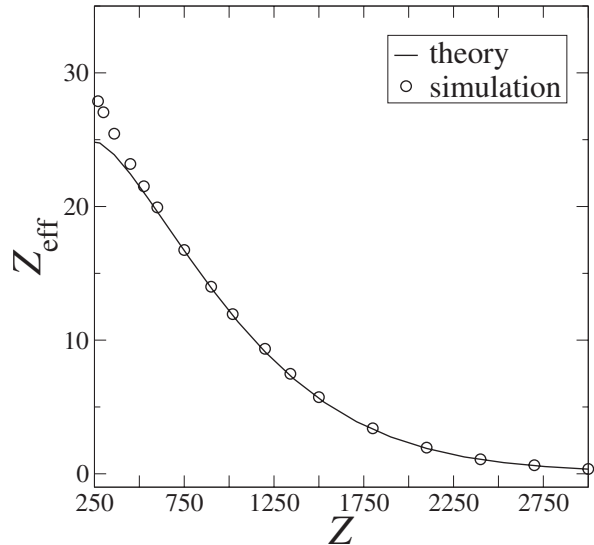

FIG. 4. The effective charge, calculated using the Alexander prescription (Ref. 5) as a function of the bare colloidal charge. For small $Z$ the theory deviates from the simulations, since the condition of Eq. (5) is violated, the agreement becomes very good for $\Gamma>4$. The trivalent counterion radius is 2 $\AA$, the colloid radius is $100 \AA$, and the WS cell is $200 \AA$.

colloidal surface form a strongly correlated fluid, ${ }^{1,12,13,17}$ - a $2 \mathrm{D}$ one component plasma (OCP), which is in thermodynamic equilibrium with the bulk electrolyte. The electrochemical potential of a counterion inside the strongly correlated sheath is

$$
\mu_{\mathrm{sc}}=\mu_{c}+k_{B} T \ln \left(\rho_{\mathrm{sc}} \Lambda^{3}\right)+\alpha q \phi(a),
$$

where $\Lambda$ is the thermal de Broglie wavelength, $\rho_{\mathrm{sc}}$ is the average concentration of condensed counterions near the colloidal surface, $\mu_{c}$ is the correlational contribution to the electrochemical potential, and $\alpha q \phi(a)$ is the mean electrostatic energy of a bound counterion. The value of $\mu_{c}$ can be approximated by that of the $2 \mathrm{D} \mathrm{OCP},{ }^{24}$

$$
\mu_{c}=k_{B} T\left(-1.65 \Gamma+2.61 \Gamma^{1 / 4}-0.26 \ln \Gamma-1.95\right) .
$$

The electrochemical potential of a bulk counterion at distance $r$ from the center of colloid is given by

$$
\mu_{\text {bulk }}(r)=k_{B} T \ln \left[\rho(r) \Lambda^{3}\right]+\alpha q \phi(r) .
$$

The condition of thermodynamic equilibrium, $\mu_{\mathrm{sc}}=\mu_{\mathrm{bulk}}$, then requires that

$$
\rho(r)=\rho_{\mathrm{sc}} \exp \left[\frac{\mu_{c}+q \alpha(\phi(a)-\phi(r))}{k_{B} T}\right] .
$$

Close to the colloidal surface, variation in the mean electrostatic energy is much smaller than $\mu_{c}$, so that Eq. (9) simplifies to

$$
\rho(\delta)=\rho_{\mathrm{sc}} e^{-\left|\mu_{c} / k_{B} T\right|},
$$

where $\rho(\delta)$ is the concentration of ions in the diffused layer, but close to the colloidal surface. The physical picture that we have in mind is that the variation in the counterion concentration occurs in three stages. First, there is an exponential decrease in the density of counterions described by the strong coupling theory, Eq. (4). In this region PB theory seriously underestimates the number of counterions, see Fig. 3 . During the second stage of the density variation, counterion concentration declines gradually to the value $\rho(\delta)$. Finally, in the third regime, the counterion distribution is gov- 
erned by the mean-field PB equation. Equation (10) allows us to relate the concentrations in the strongly correlated sheath with the concentrations in the PB regime, bypassing the complicated second stage of the density variation. Furthermore, since the PB variation in the counterion density is very gradual, following Shklovskii ${ }^{17}$ we can extrapolate it all the way to the colloidal surface, $\delta \rightarrow a+r_{c}$. The condition $\rho_{\mathrm{PB}}\left(a+r_{c}\right)=\rho_{\mathrm{sc}} \exp \left(-\left|\mu_{c} / k_{B} T\right|\right)$ at the colloidal surface thus becomes a new boundary condition for the PB equation. Again we stress that this is not the real ionic concentration at the colloidal surface, which is given by Eq. (3). Instead the value of $\rho_{\mathrm{PB}}\left(a+r_{c}\right)$ is the extrapolation of the weakly varying PB solution up to the surface of colloid. Crucial to all this discussion is the value of $\rho_{\mathrm{sc}}$, the effective concentration of condensed strongly correlated counterions. Shklovskii suggested that this could be taken to be $Z / 4 \pi a^{2} r_{c}$. As was mentioned earlier, this choice would make the theory very strongly dependent on the ionic size, see Fig. 2. It also would make it violate the contact theorem. The fundamental difficulty resides in the fact that the thermodynamic equilibrium described by Eq. (9) is established between a quasi-2D strongly correlated sheath of condensed counterions and a three dimensional bulk. While in reality the concentration of counterions near the colloidal surface varies very rapidly, the pseudo-2D OCP theory describes the chemical potential of the condensed counterions Eq. (6), in terms of the effective constant density $\rho_{\mathrm{sc}}$. This means that the three dimensional density distribution $\rho(r)$ near the colloidal surface must be coarse grained to provide the average density $\rho_{\mathrm{sc}}$. The value of $l_{\text {sc }}$ gives us a natural length scale on which the coarse graining procedure should be performed. Integrating the distribution (4), we find

$$
\rho_{\mathrm{sc}}=\frac{Z^{2} \lambda_{B} \lambda_{\mathrm{GC}}}{8 \pi l_{\mathrm{sc}}\left(a+r_{c}\right)^{4}}\left(1-e^{-l_{\mathrm{sc}} / \lambda_{\mathrm{GC}}}\right) .
$$

Substituting this into Eq. (10), we obtain the value of the diffuse counterion concentration $\rho_{\mathrm{PB}}$ which will be used as a new boundary condition at the colloidal surface for the solution of the PB equation,

$$
\rho_{\mathrm{PB}}\left(a+r_{c}\right)=\frac{Z^{2} \lambda_{B}}{(3.701) 8 \pi\left(a+r_{c}\right)^{4}} e^{-\left|\mu_{c} k_{B} T\right|} .
$$

The numerical procedure is now quite simple. The PB equation is solved iteratively with the boundary condition of vanishing electric field and electrostatic potential at the cell boundary, while the number of counterions in the cell is adjusted to give the correct effective concentration at the colloidal surface described by Eq. (12). The results of this procedure are shown as solid lines in Fig. 1. As can be seen, the agreement between the theory and the simulations is very good.

\section{CONCLUSIONS}

We have presented a general theory which allows us to accurately calculate the counterion distribution close and far away from the colloidal particle. Since the osmotic pressure and the effective colloidal charge ${ }^{5}$ are determined by the density of counterions at the WS cell boundary, the present theory is particularly suitable for their calculation. As an illustration, in Fig. 4 we show the effective colloidal charge as a function of the bare charge, calculated using the theory and compared with the results of the MC simulations. The agreement is excellent as long as the plasma parameter is sufficiently large, $\Gamma>4$.

\section{ACKNOWLEDGMENTS}

Y.L. would like to acknowledge interesting conversations with Emmanuel Trizac. This work is partially supported by $\mathrm{CNPq}$, by the National Institute of Science and Technology of Complex Fluids (CNPq/FAPESP), and by the U.S. AFOSR under Grant No. FA9550-06-1-0345.

${ }^{1}$ Y. Levin, Rep. Prog. Phys. 65, 1577 (2002).

${ }^{2}$ G. N. Patey, J. Chem. Phys. 72, 5763 (1980); L. Guldbrand, B. Jonsson, H. Wennerstrom, and P. Linse, ibid. 80, 2221 (1984).

${ }^{3}$ R. Kjellander and S. Marcelja, J. Phys. Chem. 90, 1230 (1986)

${ }^{4}$ W. B. Russel, D. A. Saville, and W. R. Schowalter, in Colloidal Dispersions, edited by G. K. Batchelor (Cambridge University Press, Cambridge, 1989).

${ }^{5}$ S. Alexander, P. M. Chaikin, P. Grant, G. J. Morales, and P. Pincus, J. Chem. Phys. 80, 5776 (1984).

${ }^{6}$ E. Trizac, L. Bocquet, M. Aubouy, and H. von Grunberg, Langmuir 19, 4027 (2003).

${ }^{7}$ R. D. Groot, J. Chem. Phys. 95, 9191 (1991).

${ }^{8}$ A. Diehl and Y. Levin, J. Chem. Phys. 121, 12100 (2004).

${ }^{9}$ A. Diehl and Y. Levin, J. Phys.: Condens. Matter 17, S3309 (2005).

${ }^{10}$ M. Deserno, C. Holm, and S. May, Macromolecules 33, 199 (2000).

${ }^{11}$ A. Martin-Molina, M. Quesada-Perez, F. Galisteo-Gonzalez, and R. Hidalgo-Alvarez, J. Phys.: Condens. Matter 15, S3475 (2003); A. Fernandez-Nieves, A. Fernandez-Barbero, F. J. de las Nieves, and B. Vincent, J. Chem. Phys. 123, 054905 (2005).

${ }^{12}$ S. Pianegonda, M. C. Barbosa, and Y. Levin, Europhys. Lett. 71, 831 (2005).

${ }^{13}$ I. Rouzina and V. Bloomfield, J. Phys. Chem. 100, 9977 (1996).

${ }^{14}$ E. Allahyarov, I. D'Amico, and H. Löwen, Phys. Rev. Lett. 81, 1334 (1998); A. W. C. Lau, P. Pincus, D. Levine, and H. A. Fertig, Phys. Rev. E 63, 051604 (2001); A. Diehl, H. A. Carmona, and Y. Levin, ibid. 64, 011804 (2001); V. Lobaskin, A. Lyubartsev, and P. Linse, ibid. 63, 020401 (2001); A. Naji and R. R. Netz, Eur. Phys. J. E 13, 43 (2004); A. A. Kornyshev and S. Leikin, Phys. Rev. Lett. 82, 4138 (1999); W. M. Gelbart, R. F. Bruinsma, P. A. Pincus, and V. A. Parsegian, Phys. Today 53(9), 38 (2000); A. A. Kornyshev, D. J. Lee, S. Leikin, and A. Wynveen, Rev. Mod. Phys. 79, 943 (2007).

${ }^{15}$ A. Y. Grosberg, T. T. Nguyen, and B. I. Shklovskii, Rev. Mod. Phys. 74, 329 (2002).

${ }^{16}$ G. M. Torrie and J. P. Valleau, J. Chem. Phys. 73, 5807 (1980); J. J. Arenzon, J. F. Stilck, and Y. Levin, Eur. Phys. J. B 12, 79 (1999); F. J. Solis and M. O. de la Cruz, Eur. Phys. J. E 4, 143 (2001); R. Messina, C. Holm, and K. Kremer, Phys. Rev. E 64, 021405 (2001); O. Lenz and C. Holm, Eur. Phys. J. E 26, 191 (2008); M. Deserno, F. Jimenez-Angeles, C. Holm, and M. Lozada-Cassou, J. Phys. Chem. B 105, 10983 (2001).

${ }^{17}$ B. I. Shklovskii, Phys. Rev. E 60, 5802 (1999).

${ }^{18}$ M. Lozada-Cassou, R. Saavedra-Barrera, and D. Henderson, J. Chem. Phys. 77, 5150 (1982).

${ }^{19}$ M. Quesada-Perez, E. Gonzales-Tovar, A. Martin-Molina, M. LozadoCassou, and R. Hidalgo-Alvarez, ChemPhysChem 4, 234 (2003).

${ }^{20}$ M. J. Stevens and M. O. Robbins, Europhys. Lett. 12, 81 (1990).

${ }^{21}$ A. Diehl, M. N. Tamashiro, M. C. Barbosa, and Y. Levin, Physica A 274, 433 (1999).

${ }^{22}$ D. Henderson and L. Blum, J. Chem. Phys. 69, 5441 (1978); H. Wennerström, B. Jönsson, and P. Linse, ibid. 76, 4665 (1982).

${ }^{23}$ A. G. Moreira and R. R. Netz, Europhys. Lett. 52, 705 (2000); A. Naji, S. Jungblut, A. G. Moreira, and R. R. Netz, Physica A 352, 131 (2005).

${ }^{24}$ H. Totsuji, J. Phys. Soc. Jpn. 39, 253 (1975). 